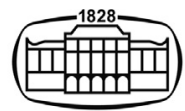

AKADÉMIAI KIADÓ

Interventional Medicine and Applied Science

$11(2019)$ 4, 216-220

Dol:

$10.1556 / 1646.2020 .00004$

(c) 2019 The Author(s)

\title{
Efficacy, safety and tolerability of bosentan as an adjuvant to sildenafil and sildenafil alone in persistant pulmonary hypertension of newborn (PPHN)
}

\author{
J.R. VIJAY KUMAR, H.S. NATRAJ SETTY* ${ }^{\circ}$, \\ M. JAYARANGANATH and C.N. MANJUNATH
}

Sri Jayadeva Institute of Cardiovascular Sciences and Research, Bangalore, Karnataka, India

Received: June 22, 2017 • Accepted: September 6, 2019

Published online: July 16, 2021

RESEARCH ARTICLE

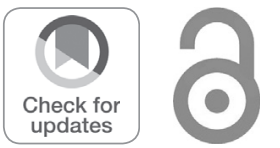

*Corresponding author. Tel.: +91080 26580051; fax: +9108022977261 (Mobile: +91 9845612322). E-mail: drnatrajsetty75@gmail.com

\begin{abstract}
Background: Pulmonary Arterial Hypertension (PAH) carries a poor prognosis in both adult and pediatric patients. It is a life-threatening condition in newborns. Current recommendations advocate the use of targeted monotherapy as a first-line approach for the treatment of Persistent Pulmonary Hypertension of the Newborn (PPHN). In case of an inadequate clinical response to treatment, an addition of a second or third agent is considered. PAH is usually managed with a phosphodiesterase 5 inhibitor or an endothelin receptor blocker. There are limited pediatric studies that address questions like which class of therapy should be initiated first or if a combination should be initiated together. With this background, the present study was initiated to compare the efficacy, safety, and tolerability of bosentan as an adjuvant to sildenafil and sildenafil alone in PPHN. Results: A total of 40 patients were enrolled in the study. Out of them, 26 were males $(65 \%)$ and 14 were females (35\%). PPHN was most commonly seen in the $29(72.5 \%)$ of participants with a history of first order birth. Mean duration of symptoms was $14.05 \pm 2.06$ days. The participants were randomized to two groups. Group A consisted of total 25 participants that received both bosentan and sildenafil and group B had 15 participants that received sildenafil alone. Both groups were comparable in terms of birth weight and present weight, consanguinity, and mode of delivery. Efficacy was determined by the reduction in mean baseline Pulmonary Artery Systolic Pressure (PASP). PASP in group A was $75.56 \pm 10.62 \mathrm{~mm} \mathrm{Hg}$ and in group B was $64.86 \pm 12.25 \mathrm{~mm} \mathrm{Hg}$ which was not statistically significant $(P>0.05)$. PASP on the third and seventh day in group A were $43.72 \pm 8.63$ and $24.47 \pm 3.52 \mathrm{~mm} \mathrm{Hg}$ compared to $42.28 \pm 9.43$ and $27.276 \pm 8.38$ respectively in group B which was statistically significant $(P<0.05)$. There were two deaths each in both groups. Two participants in Group A developed liver function abnormalities. None of the participants in Group B had adverse effects. Conclusion: Most common clinical manifestations were nonspecific. Cardiovocal syndrome was common in PPHN. We conclude that oral sildenafil treatment is a safe, simple and effective treatment for persistent pulmonary hypertension in newborn. Combination of bosentan with sildenafil is more effective and safe in reducing pulmonary artery (PA) pressures in high-risk patients with PPHN.
\end{abstract}

\section{KEYWORDS}

pulmonary hypertension, sildenafil, bosentan, cardiovocal syndrome

\section{INTRODUCTION}

Persistent Pulmonary Hypertension of the Newborn (PPHN) is a syndrome of acute respiratory failure. It is characterized by severe hypoxemia after birth, marked pulmonary hypertension, vasoreactivity with extrapulmonary right-to-left shunting of blood across the ductus arteriosus and/or foramen ovale with an absence of cyanotic congenital heart disease [1]. It is a common problem in the neonate and is associated with significant morbidity and mortality $[1,2]$. PPHN occurs in approximately 1.9 per 1,000 live births but a wide variation 
in the incidence of about $0.43-6.82$ per 1,000 live births has been reported between centers. The disease is believed to be because of an abnormal pulmonary vasoreactivity due to decreased production or responsiveness to vasodilator stimuli and/or increased production or responsiveness to vasoconstrictor stimuli. The principal goal of PPHN treatment is selective pulmonary vasodilatation. Various modalities of PPHN treatment include Ventilation strategies and pulmonary vasodilators like nitric oxide, prostacyclin analogues, phosphodiesterase inhibitors, and endothelin antagonists [3]. Although the above treatments approved for $\mathrm{PAH}$ in adults have shown favorable effects in children, pediatric treatment decisions largely depend on results from evidence-based adult studies and the experience of clinicians. Also, there are reports that mention Inhaled Nitric Oxide (iNO) or invasive treatment with Extra Corporeal Membrane Oxygenation (ECMO) as the gold standards of the PPHN therapy. But they are expensive therapeutic modalities associated with technical difficulties in developing countries. Though mortality varies from 10 to $20 \%$ of affected newborns in the developing countries, it is much higher when the therapies discussed above are not available. So alternative less expensive treatments are being sought [4].

Bosentan is a dual endothelin receptor antagonist that has been established in double-blind, placebo-controlled studies in adults with $\mathrm{PAH}[5,6]$ and in children over 3 years of age [7]. Recent studies have emphasized that oral bosentan may be a safe and effective treatment to improve oxygenation in neonates with PPHN. Sildenafil is a phosphodiesterase inhibitor type 5 (PDE5) that has been shown to selectively reduce pulmonary vascular resistance. Several case reports and controlled studies document improved oxygenation and echo cardio-graphic evidence of reduced pulmonary arterial pressures following the administration of Sildenafil therapy in newborns that had PPHN. This study was designed to assess the efficacy, safety, and tolerability of bosentan as an adjuvant to sildenafil and sildenafil alone in PPHN.

This was a prospective, randomized, interventional, open-label and comparative study. It was conducted at Sri Jayadeva Institute of cardiovascular sciences and Research, Karnataka, Bengaluru, India. It was conducted from August 2013 to January 2015 after taking approval from the Institutional Ethical Committee (IEC) of the hospital. A consecutive series of 40 patients were enrolled in this study. The inclusion criteria were as follows: i) Newborns to 3 months of age diagnosed with a known diagnosis of $\mathrm{PAH}$ before the beginning of the study or those diagnosed during the recruitment period ii) Patients with PAH Congenital Heart Disease PAH-CHD were included if increased Pulmonary Vascular Resistance (PVR) was considered unrelated to CHD iii) Informed consent by parents or Legally Authorized Representative (LAR). The exclusion criteria included children with cyanotic congenital heart disease, bleeding disorders and liver dysfunction. Patients who met the inclusion and exclusion criteria were included in the study. The diagnosis of PAH was established using Doppler echocardiography based European Society of Cardiology guidelines [8]. PAH history included the date of the first presentation, date of diagnosis, demographic profile, clinical manifestations, and functional signs. These were documented in a case report form. The participants were randomized based on computer-generated numbers [9] into Group A or Group B. Group A participants received both bosentan and sildenafil; and Group B only sildenafil. Sildenafil was administered via a nasogastric tube or orally at a starting dose of $0.5 \mathrm{mg} / \mathrm{kg}$. The dose of sildenafil was increased stepwise by $0.5 \mathrm{mg} / \mathrm{kg}$ every $4-6 \mathrm{~h}$ up to a maximum dose of $2 \mathrm{mg} / \mathrm{kg}$ with careful hemodynamic monitoring. Bosentan solution was prepared from a 125-mg tablet, which was crushed to one-fourth and then dissolved in sterile water of $10 \mathrm{~mL}(3 \mathrm{mg} / \mathrm{mL})$. Bosentan $(1 \mathrm{mg} / \mathrm{kg}$ twice a day) was administered via orogastric tube or oral. Opaque covers were used to cover the drug's container and orogastric tubes used for the administration of drugs. Hemodynamic parameters were documented at inclusion, at $3^{\text {rd }}$ day, and $7^{\text {th }}$ day after completion of treatment.

The evaluation of efficacy was based on the reduction of Pulmonary Artery Systolic Pressure (PASP) from baseline to the $3^{\text {rd }}$ and $7^{\text {th }}$ day of post-drug therapy. The safety and tolerability of the drugs were assessed by review of all study Adverse Drug Reactions (ADR). Patients were monitored for ADR like hypotension, gastric intolerance, bleeding or pulmonary hemorrhage daily during the administration of study therapy by active surveillance. Serum bilirubin, liver enzymes, alkaline phosphatase, serum creatinine, serum electrolytes and complete blood count were performed twice $3^{\text {rd }}$ and $7^{\text {th }}$ day of the drug therapy.

\section{Statistical methods}

The data were analyzed by using SAS-16.50 version. Descriptive statistics such as mean and standard deviation (SD) for continuous variables and percentage for categorical variables was determined. The reduction in PASP from baseline was evaluated using one way ANOVA followed by posthoc test. Univariate and multivariate analysis were employed to draw significant inference. A $p$-value of 0.05 or less was considered for statistical significance.

\section{RESULTS}

\section{Demographic profile}

A total of 40 participants were enrolled in the study. Out of them, 26 were males $(65 \%)$ and 14 were females $(35 \%)(P>$ $0.05)$. There were $13(32.50 \%)$ males and $12(30.0 \%)$ females in Group A, and 13 (35.20\%) males and 02 (5.0\%) females in Group B respectively. The mean age at presentation was $2.45 \pm 1.25$ years in Group A, and $2.52 \pm 1.57$ in Group B respectively. The mean birth weight of participants in Group A was $2.77 \pm 0.24 \mathrm{~kg}$, and in group B $2.65 \pm 3.02 \mathrm{~kg}$ respectively. The weight at presentation was $4.10 \pm 0.97 \mathrm{~kg}$ in Group A, and $3.85 \pm 0.84$ in Group B respectively. The gender distribution is shown in Fig. 1. 


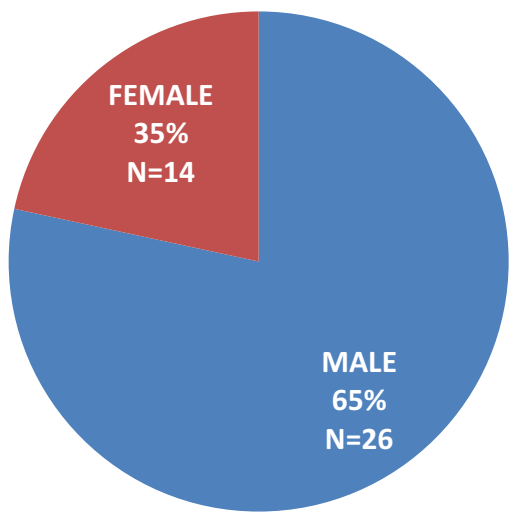

Figure 1. Gender distribution of study participants

\section{Clinical profile}

PPHN was most commonly seen in the participants that had first order of birth and the number was 29 (72.5\%).

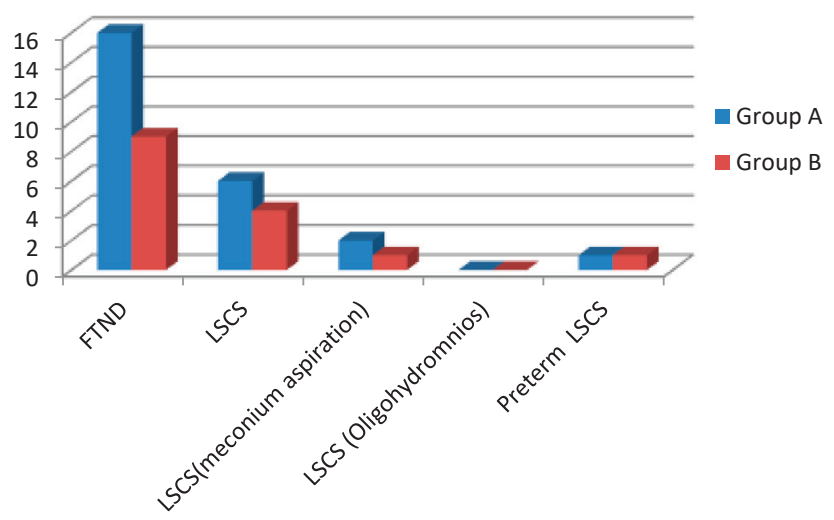

$N=40$; FTND: Full Term Normal Delivery; LSCS: Lower Segment Caesarian Section;

Figure 2. Mode of delivery of the participants
Mean duration of symptom was $14.05 \pm 2.06$ days. Regarding the mode of delivery, 25 (62.5\%) were born by Full Term Normal Delivery (FTND), 13 (32.5\%) by term Lower Segment Caesarian Section (LSCS) and 2 (5\%) were born by preterm LSCS. Consanguinity was noted in 10 (25\%) participants $(P>0.05)$. The most common clinical symptom was vomiting. It was seen in $95 \%$ participants, followed by feeding difficulty in $87.5 \%$, decrease in voice and cyanosis in $72.5 \%$, dyspnea in $60 \%$, cough in $55 \%$ and fever in $27.5 \%$ respectively. Regarding concomitant disease, hypothyroidism was noted in $2(5 \%)$ of the participants. . Tricuspid Regurgitation (TR) was trivial in $4(10 \%)$, mild in $20(50 \%)$, moderate in $10(25 \%)$ and severe in $6(15 \%)$ of the participants. Patent Foramen Ovale (PFO) with bidirectional shunt was most commonly associated lesion seen in $22(55 \%)$ of the participants. Other associated lesions seen were tiny mid muscular Ventricular Septal Defect (VSD) in 8 (20\%), 1 (2.5\%) apical VSD, 9 (22.5\%) Atrial Septal Defect (ASD), 1 (2.5\%) coronary Atrio Ventricular (AV) fistula and double aortic arch in 1 (2.5\%) patient. Right Ventricular (RV) dysfunction was seen in 15 (37.5\%) of the participants. The results are shown in Figs 2-4 and Table 1 .

\section{Efficacy}

Mean baseline PASP in group A was $75.56 \pm 10.62$ and Group B; $64.86 \pm 12.25 \mathrm{mmHg}$ respectively $(P>0.05)$. PASP on the third and seventh day in group A was $43.72 \pm 8.63$ and $24.47 \pm 3.52 \mathrm{mmHg}$ compared to $42.28 \pm 9.43$ and $27.26 \pm 8.38$ in group B respectively which was statistically significant $(P<0.05)$. Results are shown in Fig. 5.

\section{Safety}

There were two deaths each in both groups. Two participants in group A developed liver function abnormalities. None of the patients on sildenafil alone had ADR.

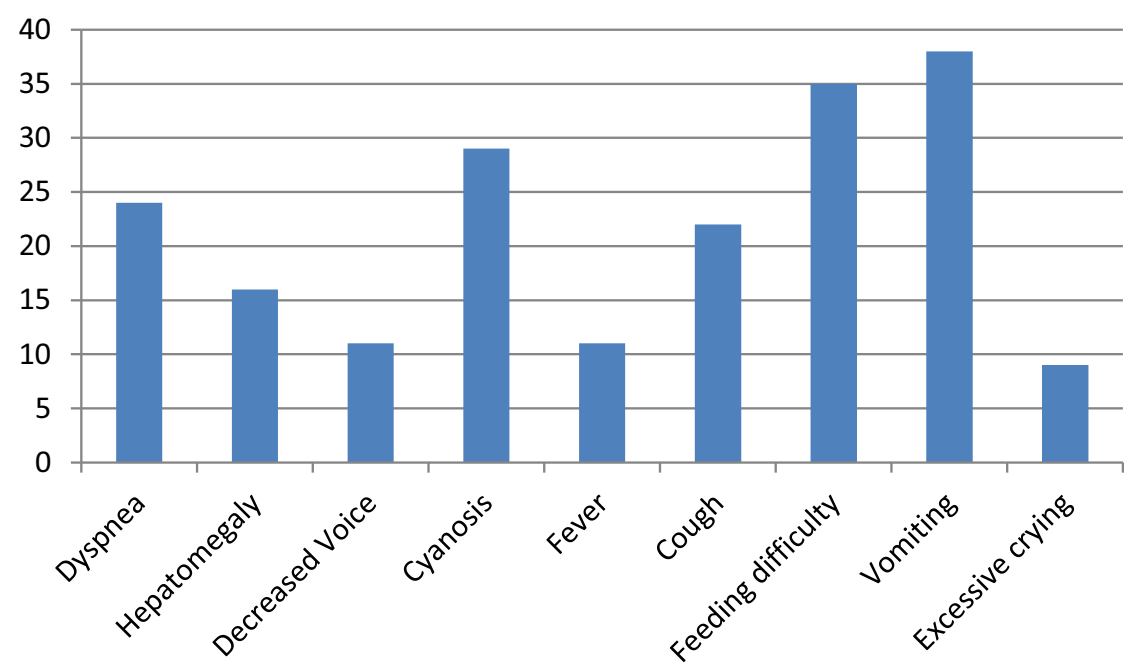

Figure 3. Clinical signs and symptoms of the participants 


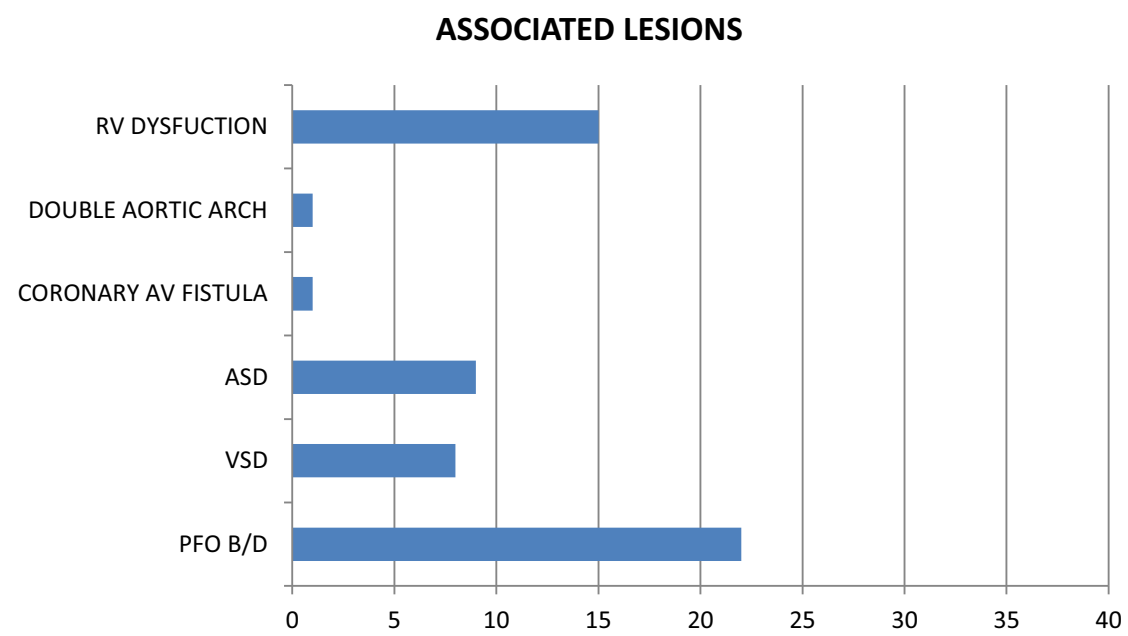

Figure 4. Associated lesions in the participants

Table 1. Distribution of tricuspid regurgitation

\begin{tabular}{lcccc}
\hline \multirow{2}{*}{ Variable } & \multicolumn{2}{c}{ Group A } & \multicolumn{2}{c}{ Group B } \\
\cline { 2 - 5 } & No & $\%$ & No & $\%$ \\
\hline TR-1 TRIVIAL & 01 & 2.50 & 03 & 7.5 \\
TR 2 MILD & 17 & 42.5 & 03 & 7.5 \\
TR3- MODERATE & 03 & 7.50 & 07 & 17.5 \\
TR4 SEVERE & 04 & 10.0 & 02 & 5.0 \\
\hline
\end{tabular}

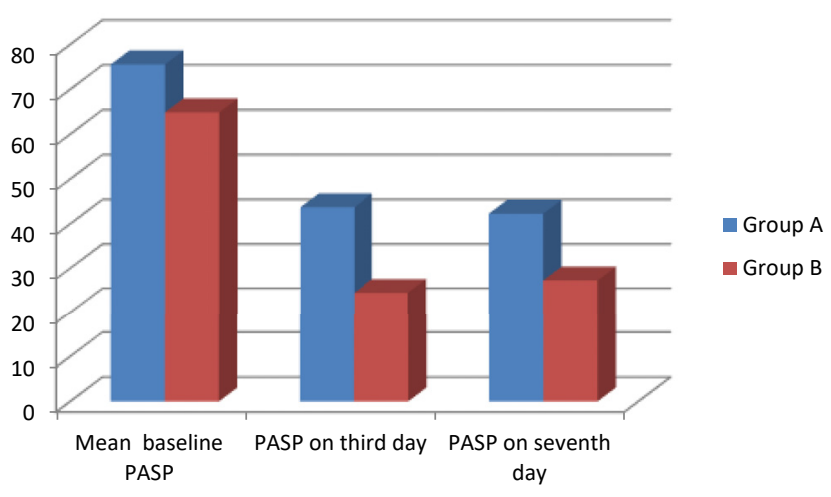

Figure 5. Comparison of efficacy

\section{DISCUSSION}

PPHN is a life-threatening condition, being among the most rapidly progressive and potentially fatal form of vasculopathy. Despite the recent advances, the clinical approach to PPHN still represents an important challenge for neonatologists. The care of newborns with PPHN requires meticulous therapeutic and ventilation strategies including, besides the stabilization of the newborn, the use of highfrequency ventilation, inhaled nitric oxide and ECMO. This prospective, randomized study was designed to assess the efficacy, safety and tolerability of bosentan in newborn infants with PPHN although not the first report use of bosentan in newborn infants with PPHN. Nakwan $\mathrm{N}$ et al. [4] reported an effective use of bosentan alone as an alternative treatment of PPHN in a full-term neonate. Goissen et al. [8] reported a successful use of bosentan as an adjunct therapy to inhaled Nitric Oxide (iNO) and sildenafil in two newborns with PPHN complicating transposition of the great arteries.

To our knowledge, this is the first study on clinical manifestations of PPHN. The most common clinical manifestations are nonspecific like vomiting, feeding difficulties, decreased voice, cyanosis, cough and fever. The peculiar symptom noted in our study was decreased voice complained by mother. We attribute it to probably dilated pulmonary artery causing compression on recurrent laryngeal nerve and trachea (cardio-vocal syndrome). Patients had improvement in voice after PA pressures were reduced. The outcome in both the groups A and B were comparable with a reduction in PA pressures and resolution of symptoms. Most of the participants on sildenafil had mild-moderate pulmonary hypertension and less of RV dysfunction compared to patients on both sildenafil and bosentan. Four deaths were seen, two in each group especially without any PFO or septal defects. This is the first prospective randomized study compared the efficacy of sildenafil and bosentan with sildenafil alone for lowering elevated PA pressure and also the clinical manifestations of PPHN.

\section{LIMITATIONS}

Our sample size is small to conclude and follow up was done only for seven days. Also, randomization was not proper as more participants in the sildenafil group had mild to moderate pulmonary hypertension compared with the combination of bosentan and sildenafil group. 


\section{CONCLUSION}

Pulmonary vasodilators are the mainstay of pulmonary arterial hypertension $(\mathrm{PAH})$ treatment. Despite all the guideline recommendations, there is always a major issue with the cost of medications, resulting in poor adherence. Our study demonstrated that treatment with bosentan along with sildenafil is safe and well well-tolerated. And that the combination is more effective and safe in reducing PAH in high-risk patients with PPHN.

Study type - Prospective Observational Study.

Study population - 40 patients with PPHN.

Study period - August 2013-January 2015.

Ethical committee - Approved.

Funding: None.

Conflict of interest: None.

\section{TYPE OF CONTRIBUTION}

Conception Dr. Vijay Kumar

Design Dr. Natraj Setty H.S

Supervision Dr. C.N Manjunath

Materials Dr. Vijay Kumar

Data collection and Processing Dr. Natraj Setty H.S

Analysis and Interpretation Dr. Jayaranganath $M$

Writer Dr. Vijay Kumar

\section{ACKNOWLEDGMENT}

N/A.

\section{ABBREVIATIONS}

PPHN Persistent Pulmonary Hypertension of Newborn

PAH Pulmonary Arterial Hypertension

PASP Pulmonary Artery Systolic Pressure

PA Pulmonary artery

iNO Inhaled Nitric Oxide

ECMO Extra Corporeal Membrane Oxygenation

IEC Institutional Ethical Committee

PVR Pulmonary Vascular Resistance

CHD Congenital Heart Disease
LAR Legally Authorized Representative

ADR Adverse Drug Reactions

SD Standard deviation

FTND Full Term Normal Delivery

LSCS Lower Segment Caesarian Section

TR Tricuspid Regurgitation

PFO Patent Foramen Ovale

VSD Ventricular Septal Defect

ASD Atrial Septal Defect

AV Atrio Ventricular

RV Right Ventricular

\section{REFERENCES}

[1] Morin, III FC, Stenmark KR. Persistent pulmonary hypertension of the newborn: state of the art. Am J Respircrit Care Med 1995;151: 2010-32.

[2] Steinhorn RH, Millard SL, Moran, III FC. Persistent pulmonary hypertension of the newborn: role of nitric oxide and endothelin in pathophysiology and treatment. ClinPerinatol 1995;22: 405-28.

[3] Konduri GG. New approaches for persistent pulmonary hypertension of newborn. ClinPerinatol 2004;31:591-611.

[4] Nakwan N, Choksuchat D, Saksawad R, Thammachote P, Nakwan $\mathrm{N}$. Successful treatment of persistent pulmonary hypertension of the newborn with bosentan. ActaPaediatr 2009;98:1683-5.

[5] Galie N, Hinderliter A, Torbicki A, Fourme T, Simonneau G, Pulido T, et al. Effects of the oral endothelin-receptor antagonist bosentan on echocardiographic and Doppler measures in patients with pulmonary arterial hypertension. J Am Collcardiol 2003;41: $1380-6$.

[6] Rubin LJ, Badesch DB, Barst RJ, Galie N, Black CM, Keogh A, et al. Bosentan therapy for pulmonary arterial hypertension. N Engl J Med 2002;346:896-903.

[7] Barst R, Ivy D, Dingemanse J, Widlitz A, Schmitt K, Doran A, et al. Pharmacokinetics, safety, and efficacity of bosentan in pediatric patients with pulmonary arterialhypertension. ClinPharmacolTher 2003;73:372-82.

[8] Goissen C, Ghyselen L, Tourneux P, Krim G, Storme L, Bou P, et al. Persistent pulmonary hypertension of the newborn with transposition of the great arteries: successful treatment with bosentan. Eur J Pediatr 2008;167:437-40.

[9] R Geggel, J Murphy, D Langleben, R Crone, J Vacanti, L Reid. Congenital diaphragmatic hernia: arterial structural changes and persistent pulmonary hypertension after surgical repair. J Pediatr 1985;107(3):457-64. https://doi.org/10.1016/s0022-3476(85)80534-5. PMID: 4032138. 